\title{
El estrecho de Magallanes como antesala del Pacífico: evolución cartográfica y toponimia entre los siglos XVI y comienzos del XVIII/
}

\author{
The Strait of Magellan as Prelude to the Pacific Ocean: \\ Cartographic Evolution and Toponymy, Sixteenth \\ to Early Eighteenth Century
}

Rodrigo Moreno Jeria

Universidad Adolfo Ibáñez, Chile

El estrecho de Magallanes fue uno de los territorios que mayor atención despertó en los cartógrafos de los siglos XVI y XVIII. De hecho, para el caso chileno, junto a Chiloé y Valdivia, el territorio magallánico es el más importante en cuanto a representación geográfica, siendo razones de navegación así como estratégicas las que explican la gran cantidad de fuentes cartográficas existentes en archivos públicos y privados nacionales como extranjeros. El presente estudio da a conocer una nueva pieza que forma parte de un derrotero que se conserva en el Archivo de la Marina de Guerra del Perú, cuya datación aproximada es de comienzos del siglo XVII, aunque con representaciones geográficas del siglo anterior.

Palabras Clave: Estrecho de Magallanes; Cartografía; Derrotero; Narborough; Dampier.

The Strait of Magellan was one of the geographical areas which generated more attention in the cartographers during the XVI and XVII centuries. In fact, for the Chilean case, together with Chiloe and Valdivia, the Magellanic region is the most represented. The reasons are both navigational and strategic purposes. That means that the surviving examples on public and private archives of several countries are comparatively abundant. The present study shows a new piece, which forms part of a navigational chart or «Derrotero» that is conserved in the Historical Archive of the Peruvian Navy, whose dating is roughly the early XVII century, although showing geographical representations from the previous century.

KEYwords: Strait of Magellan; Cartography; Derrotero; Narborough; Dampier. 
Magallanes es el territorio del que mayor documentación cartográfica colonial se conserva en Chile. De hecho, tras el pionero y clásico Ensayo acerca de una mapoteca chilena, de José Toribio Medina, ${ }^{1}$ en los tiempos modernos, el primer estudio a modo de corpus monumental que se realizó en dicho país fue la magnífica Cartografía Magallánica 1523-1945, publicada por Mateo Martinic en $1999,{ }^{2}$ la cual, junto con poner justicia a la importancia de dicho territorio en la historia, dio el impulso necesario para que tras esta obra otras investigaciones continuaran el derrotero de buscar, catastrar y poner en valor la documentación cartográfica existente para territorios de alto valor estratégico como Chiloé y Valdivia. ${ }^{3}$

Ahora bien, la travesía de Magallanes en 1520, una década y media antes de que las primeras exploraciones marítimas recorrieran Chile central, permitió identificar y representar el territorio austral del recién descubierto Océano Pacífico casi un cuarto de siglo antes de que el resto del territorio comenzara tímidamente a ser incluido en la cartografía del Nuevo Mundo que avanzaba desde el norte hacía el sur. ${ }^{4} \mathrm{Si}$ bien el mapa esquemático de Antonio Pigafetta, podría ser considerado el primer antecedente cartográfico del descubrimiento del estrecho, ${ }^{5}$ de seguro hubo bosquejos confeccionados por los pilotos de la referida expedición, sin embargo, como fue frecuente en la historia de la cartografía náutica, dichos apuntes y notas no llegaron hasta nosotros. No obstante, una pieza atribuida a Sebastián Elcano, posiblemente realizada tras su viaje, sería con el dibujo de Pigafetta la representación preliminar más antigua del estrecho de Magallanes. ${ }^{6}$

En este sentido, y siguiendo a Martinic, terminada la empresa de Magallanes-Elcano, el primer mapa que hace alusión concreta al estrecho se realizó en 1523, es decir, apenas tres años después del magno descubrimiento. El documento está inserto en el planisferio denominado Padrón Real de Turín, de autor desconocido, y que se conserva en la Biblioteca Real de dicha ciudad. ${ }^{7}$ Esta obra, que es copia de una realizada en Sevilla

1 Medina, 1889.

2 Martinic, 1999.

3 Hay varios trabajos publicados en los últimos 30 años, aunque ninguno pretendía reunir o catastrar en la medida de las posibilidades investigativas, toda la información respectiva a un territorio específico. En esa categoría se inserta el de Guarda, 1981. También el de Vásquez, 1999. Con respecto a trabajos en la línea de Martinic, Guarda y Moreno, 2008; Guarda y Moreno, 2010; Rosenblitt y Sanhueza, 2010.

4 Biblioteca Nacional de Viena, Carta Universal de Sancho Gutierrez, 1551, 108 x $336 \mathrm{~cm}$

5 Pigafetta, 1922, 145

6 Martinic, 1999, 18. 
por orden de la Casa de la Contratación, recogió con bastante precisión la información de los sobrevivientes de la expedición que regresaron a la capital andaluza el 9 de septiembre de $1522 .{ }^{8}$

Desde aquel momento, fueron numerosas las representaciones del estrecho en la cartografía del siglo XVI, llegando a un número superior al centenar las cartas y mapas de un territorio de alto valor estratégico para la navegación hispana hacia Oriente, así como la conexión natural hacia la costa americana del Pacífico sur y norte de los dominios castellanos. Sin embargo, estas representaciones cartográficas no coincidieron con un período de exhaustiva investigación de las costas y de la realidad geográfica del paso austral, siendo en muchos casos, una simple idealización de un estrecho que separaba el Nuevo Mundo de la mítica Terra Australis. De hecho, desde el Atlántico, sólo se registran hasta la mitad del siglo XVI cuatro expediciones españolas con suerte dispar, siendo la del obispo de Plasencia, liderada por Francisco de Camargo en 1540, la única que entregó algunas noticias complementarias al viaje de Magallanes, pero en ningún caso relevantes para realizar nuevos levantamientos geográficos de interés. ${ }^{9}$ Es decir, el camino al Mar del Sur o Pacífico, si bien fue representado cartográficamente a partir de la gesta de Magallanes y su expedición, poco se hizo por avanzar en el conocimiento de un paso que requería un buen soporte cartográfico para poder sortearlo con éxito.

Tampoco ayudó en demasía a la historia de la cartografía magallánica los intentos que se hicieron a partir de 1553 para explorar la costa occidental austral desde la gobernación de Chile, y que permitió la navegación de Hernando Gallego primero y luego la de Juan de Ladrillero en 1558, sin embargo, algunas noticias de ambas expediciones quedaron reflejadas en la cartografía que por entonces era elaborada en Sevilla. Así al menos queda demostrado con la influencia de las noticias de Ladrillero en las correcciones al mapa de Sancho Gutiérrez confeccionado inicialmente en $1551 .^{10}$

Desde la perspectiva española, la expedición que en el siglo XVI realizó la más importante contribución cartográfica del estrecho fue la de Pedro Sarmiento de Gamboa quien, en su viaje de exploración de 1579 y

7 Martin, 1993, 89.

8 Martinic da una posible atribución a Diego Ribero, siempre y cuando el plano se hubiese confeccionado en Sevilla a principios de 1523. Martinic, 1999, 18.

9 Ibidem, 11. Fue con el naufragio del capitán Argüello y sus compañeros, en que se dio inicio al clásico imaginario de la Ciudad de los Césares.

10 Martin, 1999, 112. 
1580, y luego en su proyecto fundacional, realizó importantes aportes al conocimiento detallado de las costas de dicho paso austral, tal como se señala en la edición española de su viaje, «La descripción que hace del Estrecho de Magallanes se contempla mui instructiva y útil a los navegantes, porque no solo da la configuración de los Montes, sino que señala mui prolixamente las corrientes, sondas, Ríos, Ensenadas y Puertos que en él se hallan, informando de los parages que vio poblados, como de sus moradores». ${ }^{11}$ Lamentablemente, tampoco fue mucho lo que se pudo aportar a la cartografía del momento, como se confirmaría en la edición del viaje de Sarmiento de 1768, aún en aquella época se tenía noticia de que existían «cartas de marear que formó el mismo Sarmiento, pero por más diligencias que se han hecho, no se ha podido descubrir su paradero». ${ }^{12}$

Dado lo anterior, para la historia de la cartografía, trascendieron de mejor forma las figuras de los navegantes ingleses Francis Drake en 1578 y Thomas Cavendish en 1587, quienes demostraron que el hito magallánico tenía alta importancia para las potencias emergentes que estaban prontas a disputar la supremacía del Pacífico al Imperio español, y cuyas noticias se divulgaron con mayor facilidad entre los cosmógrafos de su tiempo.

En el caso de Drake, la expedición si bien no realizó una exhaustiva exploración geográfica del estrecho, dado la rapidez con que surcó el paso en tan solo diecisiete días, aun así legó de manera significativa a la toponimia magallánica la isla de Elisabeth y los islotes vecinos de St. Georges and St. Bartholomew, entre otras referencias que perduraron en el tiempo. ${ }^{13}$ Además, tras la proeza en el estrecho, su hallazgo más significativo fue el cruce por el sur de Tierra del Fuego, el que si bien no pudo surcar de occidente a oriente, su referencia marcó la toponimia cartográfica del futuro. Lamentablemente no quedaron vestigios cartográficos de manos del avezado navegante, aunque los testimonios de su paso al Mar del Sur fueron representados por la cartografía del siglo XVII y, además, fueron referidos en el relato del Francis Fletcher acerca del viaje, sumando un mapamundi que puso en evidencia no sólo la travesía del estrecho y el descubrimiento del paso por el sur, sino toda la circunnavegación que, por segunda vez en la historia de Occidente, realizaba un navegante. Además, era la primera ocasión que un inglés surcaba las aguas del Pacífico. ${ }^{14}$

11 Sarmiento de Gamboa, 1768, VII. Las palabras son del editor de la obra.

12 Ibidem, XXXVII.

13 Fletcher, 1628, 35; Cummins, 1995, 87; Martinic, 1999, 39.

14 En la Universidad de Yale se conserva un mapa atribuido a la expedición. 
En cuanto a la expedición de Cavendish, inglés célebre por el hallazgo de los restos de la ciudad del Rey Don Felipe, quedó de manifiesto la voluntad del navegante por conocer en profundidad el territorio y perpetuar la memoria de su exploración del espacio geográfico. De ahí que en dicha empresa realizada en 1587 legase importantes topónimos a la geografía magallánica, algunos de los cuales hoy perduran con gran relevancia como, por ejemplo, Port Famine, ${ }^{15}$ el sitio donde estaba emplazada la fallida fundación de Sarmiento; Froward ${ }^{16}$ el estratégico cabo occidental que antes se llamaba Morro de Santa Águeda, y Port Gallant, la caleta en la ribera norte del estrecho. ${ }^{17}$ Estos topónimos se conocieron por primera vez en un mapa del estrecho datado en 1588, siendo hasta la fecha el único mapa del territorio atribuido a este corsario inglés. ${ }^{18} \mathrm{El}$ propio Cavendish intentó una segunda empresa en abril de 1592 sin mucha fortuna, pues se volvió a encontrar con los hitos geográficas aludidos, aunque esta vez no pudo cruzar a la boca occidental, viéndose obligado a regresar a Europa falleció en la travesía en el verano de dicho año.

Muchas otras expediciones extranjeras fueron las que surcaron las aguas del estrecho, entre ellas la del corsario inglés Richard Hawkins, quien exploró al sur del cabo Froward, o la del neerlandés Simón de Cordes, quién bautizó la bahía de Ridder y la que lleva su nombre. ${ }^{19}$ En esta última expedición, destacó el trabajo realizado por el piloto Jan Outghersz, quien tras nueves meses de permanencia en el estrecho hizo grandes contribuciones a la cartografía, en especial, con la primera carta particular de Magallanes, titulada Fretum Magellanicum y publicada más tarde en varias ediciones. ${ }^{20}$ De igual forma, el almirante Olivier van Noort, también incursionó en el estrecho, cuya expedición dejaría huella por ser el primer neerlandés en circunnavegar el mundo y el cuarto tras los antes referidos Magallanes-Elcano, Drake y Cavendish. El viaje de van Noort, que no estuvo exento de sobresaltos, finalizó el 1601, tres años después de haber

15 El nombre, dado por Cavendish el 6 de enero de 1587, perduró en el tiempo en su traducción castellana de Puerto del Hambre. Véase Barros, 1978, 65-75 y Espinoza, 2010, 1456-1460.

16 El cabo Froward, «hostil» o «rebelde», se llamó anteriormente Morro de Santa Águeda, bautizado por Sarmiento de Gamboa en 1580, sin embargo, el nombre dado por el inglés, perduró en el tiempo.

17 Gallant era el nombre de la tercera nave de la expedición de Cavendish, y el lugar fue bautizado el 21 de enero de 1587.

18 El mapa se titula Kaart van de Westkust van Zuid-Amerika, Van de Straat Magellaan tot aan Nueva Espania. Véase Martinic, 1999, 199.

19 Ibidem, 50. También en Alcedo, 1788, .420.

20 Martinic, 1999, 57-58. 
iniciado la campaña y solo con una nave de las cuatro que habían inicialmente emprendido la travesía. Felizmente este viaje dejaría información en cuanto al paso por Magallanes, lo que permitió a cartógrafos y editores holandeses representar el mundo austral y el estrecho en específico sobre una serie de mapas de la época.

A propósito de lo anterior, en el siglo XVII se experimenta un aumento de la producción cartográfica del territorio magallánico, coincidente con la importancia que va adquiriendo el estrecho en la navegación ultramarina española, así como de las potencias emergentes. De igual forma, los importantes descubrimientos del estrecho de Le Maire y el cabo de Hornos, a partir de la expedición de Schouten y Le Maire en 1616, dieron aún mayor realce a la navegación austral. De hecho, este hallazgo, desde la óptica de la cartografía, en nada afectó el interés por el estrecho de Magallanes, porque el nuevo descubrimiento de los neerlandeses se enmarcaba en el entorno magallánico y de la Tierra del Fuego y, por lo tanto, las nuevas representaciones reemplazaron paulatinamente la infaltable Terra Australis por una Tierra del Fuego circunnavegable. Esto se hizo de manera especial con el viaje de los hermanos Nodal, cuyo relato y representación cartográfica, delineada por Pedro de Teixeira de Albernaz y grabada por Juan de Courbes, apareció publicada en 1621, rebautizando el paso Le Maire como de San Vicente y a Hornos como San Ildefonso, nueva toponimia que no prosperó en la cartografía de su tiempo. ${ }^{21}$

Entre las expediciones que continuaron en el siglo XVII, fuera de las ya mencionadas para el caso del cabo de Hornos, la del almirante Joris van Spilbergen en 1615 tuvo gran importancia para la navegación ultramarina por tratarse del segundo neerlandés que concretó la circunnavegación al globo. En cuanto a su recorrido por el estrecho de Magallanes, un plano de 1619 dejó testimonio de su paso por las islas de los Pingüinos, parada habitual para los marinos de su tiempo que buscaban allí reaprovisionamiento, así como la incorporación de nueva toponimia en el sector noroccidental. ${ }^{22}$

Posteriormente, otras expediciones surcaron las aguas australes aunque pasaron al Pacífico por el referido cabo de Hornos. Tal fue el caso del Almirante neerlandés Jacobus L'Hermite, quien realizó importantes reconocimientos geográficos en el entorno del mencionado cabo, descubriendo

21 Nodal, 1621, 28-40.

22 Tijpus Freti Magellanici quod Giorgius Spilbergius cum clase lustravit (1619). Martinic, 2012, 7-22. 


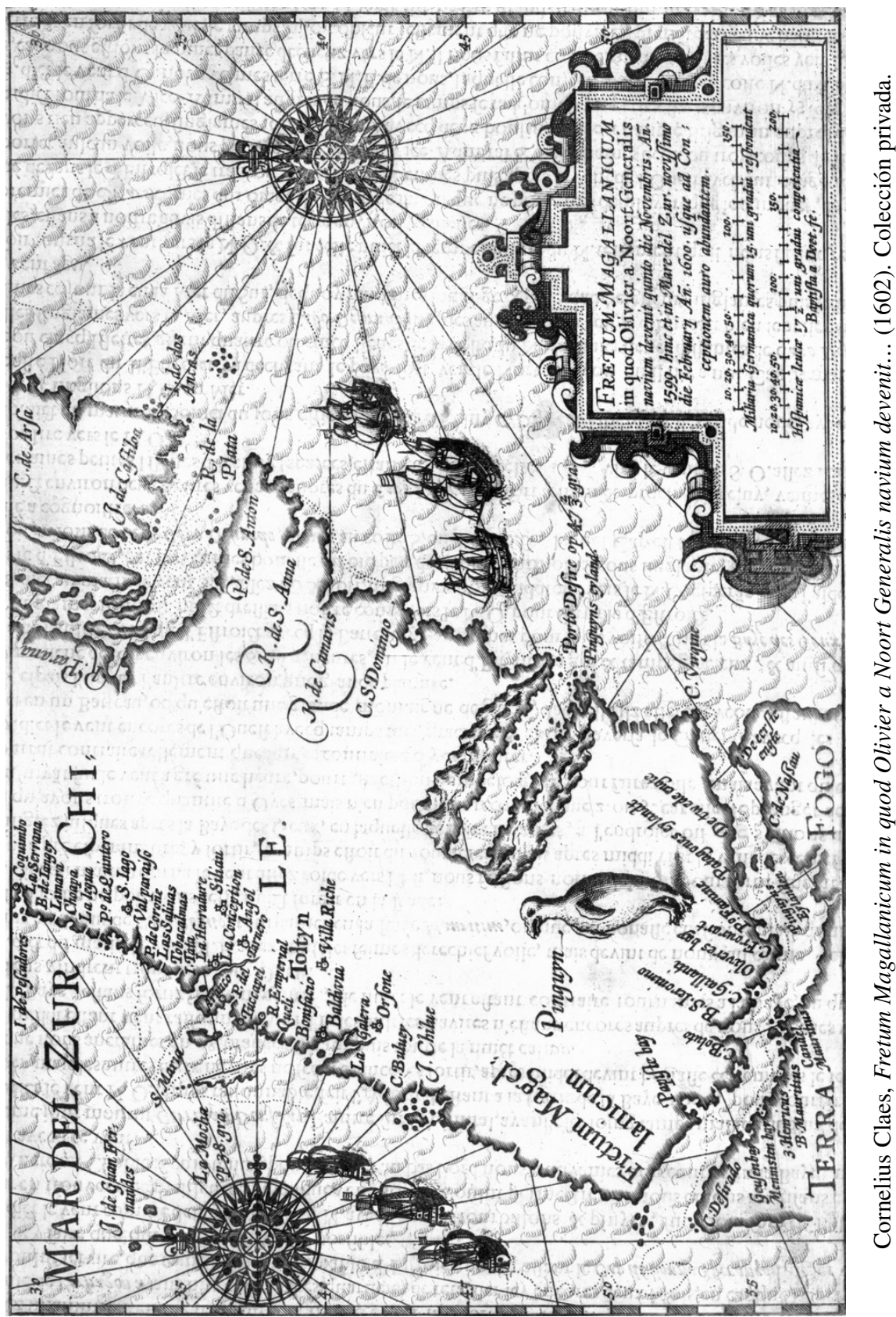




\section{RODRIGO MORENO JERIA}

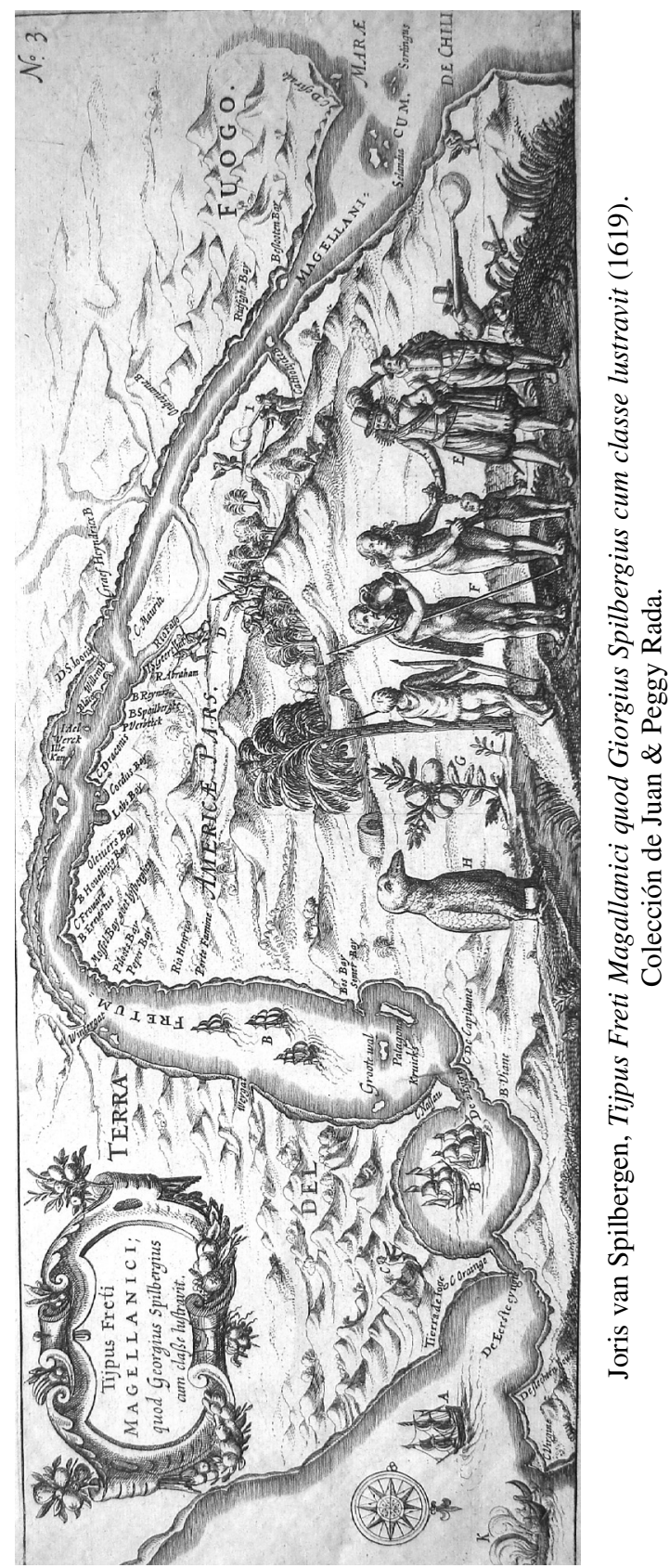


y bautizando el golfo de Nassau y explorando las islas del sur del actual canal Beagle. Avanzando en el siglo, el estrecho de Magallanes había sido sembrado con importantes topónimos que permanecieron a través de tiempo como parte del conocimiento cartográfico, sin embargo, la navegación por el mismo se había hecho poco habitual, siendo evidente el interés que despertaba en los marinos de su tiempo el paso por el sur, como alternativa más segura y rápida.

No obstante lo anterior, fue en 1670, con el arribo del capitán John Narborough cuando nuevamente el estrecho cobró más importancia, puesto que esta vez el nuevo expedicionario tenía por misión hacer un levantamiento cartográfico e hidrográfico del paso, con el fin de posibilitar la navegación futura de las naves británicas, y la posible toma de posesión del mismo. El capitán John Narborough era un inglés originario de Norfolk que, antes de realizar sus incursiones por los mares australes, había sido héroe en la segunda guerra anglo-holandesa, entre 1665 y 1667. Comisionado por el almirantazgo, asumió la misión de emprender una travesía al estrecho de Magallanes y a la costa del virreinato del Perú con dos naves, el navío Sweeptakes y el pingüe Bathelor, cuyo objetivo era entablar posibles relaciones económicas, además de hacer reconocimiento de los territorios australes y la costa del Pacífico sur de América. ${ }^{23}$ De hecho, las instrucciones recibidas dejaban en claro los fines pacíficos de la empresa: «No debía tocar puertos ni poner gente en tierra, a menos que hubiera una necesidad apremiante, hasta que avanzara más allá del Río de la Plata, y que hiciese daño a ningún español con quien se encontrara ni se metiera en cualquier lugar que tuviese poblado». ${ }^{24}$

La expedición zarpó en septiembre de 1669 desde Inglaterra y, tras haber hecho un reconocimiento de la costa oriental patagónica, específicamente en Puerto Deseado y San Julián, arribó a Magallanes el 22 de octubre de 1670. Para entonces, ya se había perdido contacto con el pingüe, por lo que el Sweeptakes quedó como la exclusiva embarcación de la empresa. Durante un poco más de un mes, Narborough dispuso un reconocimiento exhaustivo de Magallanes, realizando sondajes y elaborando una carta de navegación que sin duda sería de mucha utilidad para las futuras navegaciones. Mientras duró su estadía se bautizaron algunos hitos geográficos, como por ejemplo, Sandy Point, que para entonces aún era conocido como

23 Vargas, 1788, 264.

24 Bradley, 1992, 267.

Anu. estud. am., 70, 2, julio-diciembre, 2013, 419-439. ISSN: 0210-5810. DOI: 10.3989/aeamer.2013.2.02 
cabo de San Antonio, según la expedición de Sarmiento, y que desde ahora se impondría sin mayores cuestionamientos, a tal punto que con los años el lugar terminó por castellanizarse como Punta Arenas. De igual forma, la bahía Oosy u Oasy fue bautizada por el navegante inglés, topónimo que perdura hasta nuestros días, junto a otros ejemplos de la toponimia magallánica..$^{25}$

Tras un mes de exhaustiva exploración, así como de contacto con indígenas del lugar, el capitán inglés pudo realizar un notable levantamiento cartográfico con los referidos sondajes, mediciones e identificación de fondeaderos. ${ }^{26}$ Narborough abandonó el paso por la boca occidental el 26 de noviembre de 1670, continuando el viaje hasta Valdivia, lugar donde finalmente se vio interrumpido el proyecto original dado que las autoridades locales retuvieron a algunos tripulantes de la expedición, entre ellos un español, que habían descendido a tierra pacíficamente. La incomprensión y sospecha de la misión del inglés hacía impracticable el éxito de la empresa en cuanto a los objetivos iniciales, por lo que Narborough decidió poner fin a la misma y emprender rumbo nuevamente hacia Inglaterra. ${ }^{27}$ Valga sí hacer notar que al regreso, queda de manifiesto en los escritos del navegante, que si bien la misión era exploratoria, no era estrictamente pacífica en sus intenciones futuras, puesto que se planeaba tomar posesión efectiva de tierras magallánicas y de Valdivia, ciudad que tenía fama por sus riquezas auríferas cercanas. ${ }^{28}$

$\mathrm{Al}$ regreso, Narborough elaboró los informes y sus convicciones acerca del futuro de Inglaterra en Magallanes y la costa sur de Chile, para lo cual concluyó satisfactoriamente planos realizados en el estrecho y que se convirtieron en la mayor realización cartográfica de su tiempo. Aunque hubo omisiones e imperfecciones, en especial en la costa noroccidental fueguina, los levantamientos de Narborough así como su vasta toponimia que llegó a cuarenta nombres que Martinic y Moore identifican en una acuciosa investigación realizada en 1982, fue un aporte insuperable para la época. Una parte no menor de los topónimos sobrevivió al tiempo, a las futuras navegaciones y a los trabajos hidrográficos. ${ }^{29}$ Son tres los mapas manuscritos específicos que se conservan sobre el estrecho y que provie-

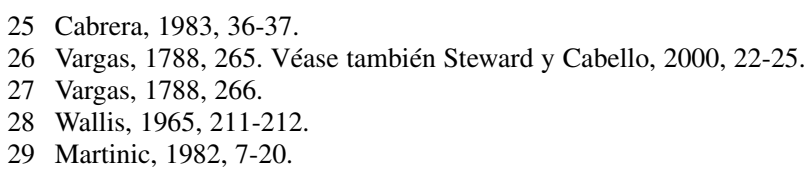


nen de la expedición de Narborough. Sin embargo, recientemente se han realizado nuevos hallazgos de documentos y cartografía relativos a su expedición, lo que abrirá nuevas perspectivas del viaje y de sus consideraciones técnicas. ${ }^{30}$ Con relación a las cartas manuscritas del estrecho, John Thorton realizó la impresión de un mapa del estrecho con las noticias geográficas e hidrográficas en 1671 que rápidamente se dio a conocer en los círculos cartográficos y marítimos. Para entonces España ya tenía conocimiento de dicho viaje y de las importantes indagaciones que había hecho Narborough en Magallanes, así como una peligrosa toma de posesión tanto de la costa patagónica como de Tierra del Fuego. De allí que surgió la necesidad de realizar incursiones hispanas desde el virreinato del Perú para marcar soberanía sobre un territorio y paso austral que había tenido poco uso en una buena parte del siglo.

En cuanto al plano publicado, que se titula A New Mapp of Magellan Straights discovered by cap. John Narbrough (sic), éste representa en detalle la geografía del estrecho con un notable progreso con respecto a lo que se conocía a la fecha. Además, agregó un mapa del extremo sur de América para que se pudiese comprender y contextualizar geográficamente el hito particular que era el paso de Magallanes en toda su dimensión. Fuera del anterior, y junto con aportar la referida toponimia, incluye elementos de análisis de gran interés como un bosquejo del tipo de chozas donde habitaban los aborígenes en la Patagonia, así como también, especies animales propias del territorio, como el guanaco y el ñandú, noticias de alto valor científico que rompen la decorativa imaginaria presente hasta el siglo XVII. ${ }^{31}$

Posterior a la empresa de Narborough, los españoles intentaron consolidar la dominación del estrecho, con las expediciones de Bartolomé Díaz Gallardo en 1674, Antonio de Vea y el propio Díaz en 1675, y Pascual Uriarte en el mismo año, todos desde Chiloé. ${ }^{32}$ Sin embargo, ninguno pudo arribar con éxito a Magallanes aunque sí quedó testimonio cartográfico en el Derrotero del Mar del Sur, que actualmente se conserva en la Biblioteca Nacional de Perú en Lima el cual, si bien está datado en 1730, contiene mapas de 1676, fruto de las duras incursiones realizadas por los canales

30 La British Library ha anunciado recientemente la incorporación de nuevos documentos a la colección que incluyen entre otros, mapas de Puerto Deseado y Valdivia.

31 John Thorton, A New Mapp of Magellan Straights discovered by cap. John Narbrough, Londres 1671. Colección privada.

32 Martinic, 1999, 86. 
RODRIGO MORENO JERIA

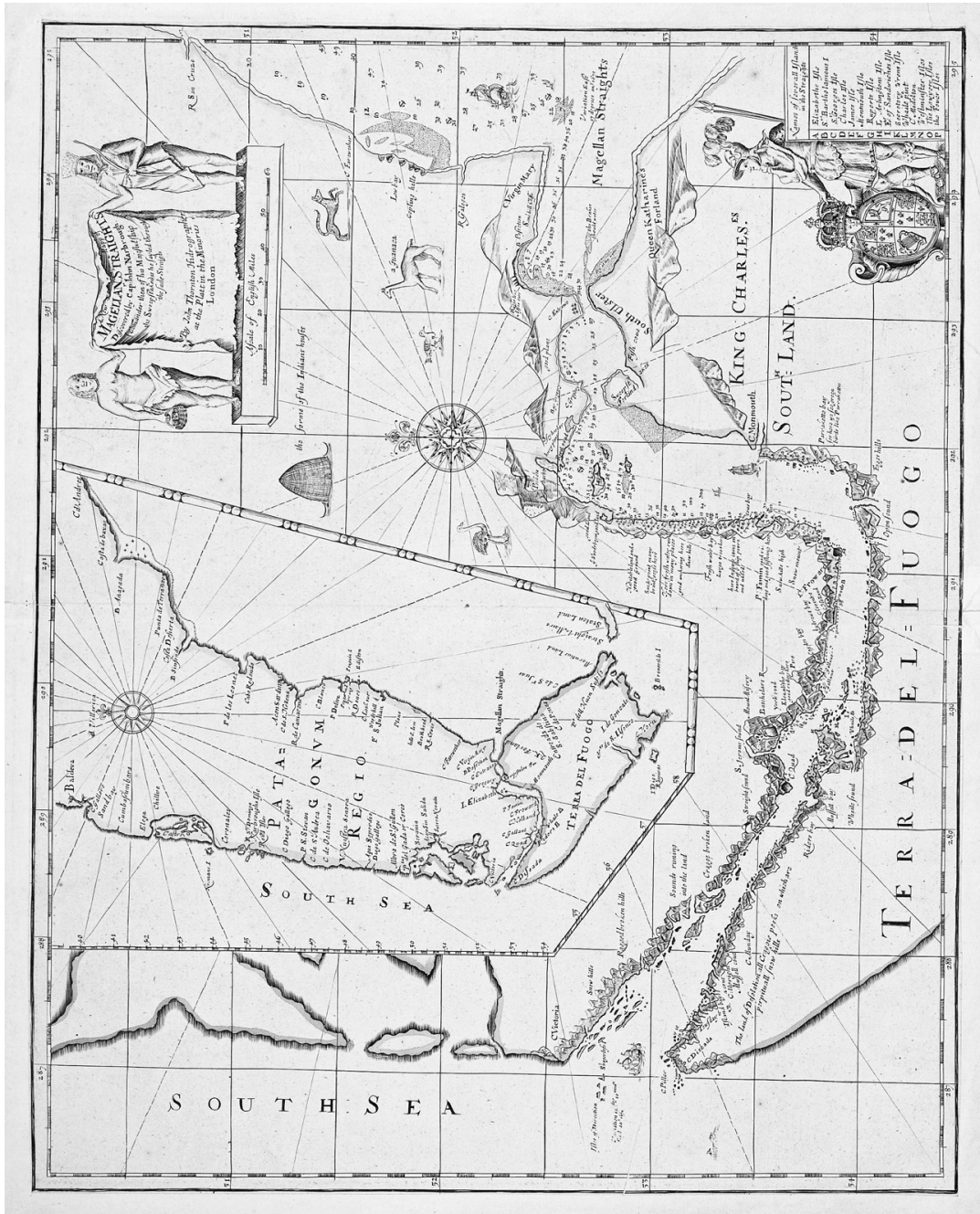

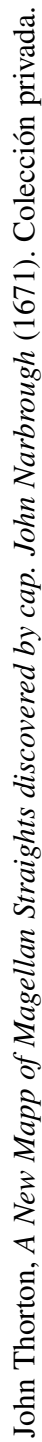


australes rumbo a Magallanes. ${ }^{33}$ En1690 se pudo conocer un nuevo levantamiento cartográfico realizado por Francisco de Seixas y Lobera que, aunque adjunta importante información geográfica y de navegación, no hace un aporte significativo a la representación cartográfica. ${ }^{34}$

En los últimos años del siglo XVII no hubo mucha actividad en cuanto a la exploración y navegación en el estrecho, Si bien se conoce la presencia de piratas de origen inglés y francés, poco se pudo sumar a lo que tan brillantemente había realizado Narborough. Diversos vestigios toponímicos evidencian que algunos navegantes hicieron incursiones o fondearon en algunos puntos determinados en el estrecho. Por ejemplo, se constata la presencia de franceses en la bahía de Cordes en 1694 y 1695. ${ }^{35}$ Si bien hubo otras navegaciones y exploraciones menores por el estrecho en los últimos años del siglo, la ausencia de navegaciones exploratorias más exhaustivas no significó una merma en la producción cartográfica, pero ésta continuó repitiendo o complementando la información que, hasta fines de siglo, circulaba en los centros de conocimientos e información, principalmente en los territorios que coincidentemente disputaban a España la supremacía del océano Pacífico, es decir, Inglaterra y Francia.

Frente a este escenario, resulta de sumo interés la cartografía manuscrita que aún se conserva para este período, contenida en los derroteros náuticos que, dispersos en diversos repositorios contienen valiosa información sobre el estado del conocimiento magallánico a principios del siglo XVIII. El análisis de uno de estos corpus documentales y cartográficos, concretamente, para el caso del estrecho de Magallanes, permite observar de qué manera los viajes y exploraciones realizados en los siglos XVI y XVII, en especial los trabajos de Narborough, influyeron en las cartas manuscritas que los pilotos utilizaban en sus viajes por el referido estrecho y de qué forma había evolucionado la compleja toponimia del territorio.

El derrotero conocido como Derrotero Inglés, resguardado hasta hoy en el Archivo Histórico de la Marina de Guerra del Perú, es una de las joyas cartográficas más importantes conservadas en América del Sur y que sólo fue conocido por el mundo académico hispano a partir de 1988, cuando Jorge Ortiz Sotelo lo publicó en su obra Un Derrotero Inglés de las Costas

33 Ortiz Sotelo, 1993. Guarda y Moreno, 2008, 79-82. La carta que representa el estrecho es la más precaria, prueba de que no pudieron alcanzarlo, y por lo tanto, se basaron en mapas que circulaban a mediados de siglo en los derroteros españoles.

34 Seixas y Lobera, 1690, 60-79.

35 Martinic, 1999, 307-320. 
de América (1703-1704). ${ }^{36}$ La existencia documentada de este manuscrito se remontaba a la segunda mitad del siglo XIX cuando el médico escocés Jacob Dickson Hunter, residente en Arequipa, lo adquirió en fecha y lugar no precisado con exactitud. ${ }^{37}$ Posteriormente esta fuente pasó a manos de Edmundo Escomel, amigo de Hunter, quien a su vez lo donó al Presidente Augusto Leguía en 1925. Éste lo dejó en manos de la Marina de Guerra del Perú, que lo conservó en el Museo de la Institución al interior de la Escuela Naval, aunque en 1981 pasó a formar parte del Archivo Histórico de la Marina, en San Isidro, Lima, donde actualmente se conserva. ${ }^{38}$

El manuscrito consta de 58 fojas por verso y reverso, representa la costa del norte de México, específicamente Acapulco, hasta el Río de la Plata, incluyendo una carta general de la costa señalada, y está acompañado de instrucciones de navegación, todo en ello en inglés. ${ }^{39}$ En cuanto al estrecho de Magallanes, tema de nuestro interés, se presenta una carta completa y detallada que ocupa las fojas 46r y 47 y, que a diferencia de todas las otras bahías y puertos, el detalle de la representación es, para su tiempo, muy completo y detallado. Inspirándose claramente en la carta de Narborough de 1670, tiene el valor de que, en vez de haber simplemente incorporado aquella al derrotero, se delinea de la misma forma y estilo que el resto de las cartas que componen el corpus general, lo cual demuestra que el autor tuvo en su mano noticias directas del capitán inglés, pero que complementó con la propia experiencia de la navegación, cuya síntesis era los derroteros que pretendían ser referencias efectivas para la navegación segura en el Mar del Sur y la costa patagónica oriental.

En cuanto a la fecha de elaboración del derrotero, por la propia influencia de Narborough, quien navegó por mares australes en 1669, y por referencias posteriores a la navegación de Bartolomé Sharp y Basil Ringrose de 1682 y la de Jacques Gouin de Beauchene en 1701, la datación que se ha propuesto hasta la fecha es de 1702 ó 1703, aunque es claro que al derrotero se le agregó información posterior, como por ejemplo, las variaciones magnéticas indicadas en el documento, que corresponden, en algunos casos, a los años 1703 y 1704. Como bien identificó Ortiz Sotelo, el uso de otra tinta en dicha información así como también en otras hojas con algunos detalles de la información geográfica, hacen pensar que este

36 Ortiz Sotelo, 1988.

37 Se habla que pudo ser adquirido en Mollendo.

38 Ortiz Sotelo, 1988, 11

39 Archivo Histórico de la Marina de Guerra del Perú (AHMGP), Fondo Derrotero Inglés, 7. 
documento fue intervenido durante su uso en la navegación, al menos hasta 1707, fecha en que dejó de usarse por naufragio o captura.

Ahora bien, hay que agregar a ello, otro elemento de interés en relación con el conocimiento geográfico de las costas del Pacífico y en especial con las del extremo meridional. Ahí claramente se observa que las cartas relativas a Valdivia y a Chiloé corresponden a la cartografía de la segunda mitad del siglo XVII. Por lo que es altamente probable que en el derrotero, al ser una sumatoria de experiencias de navegación comentadas por el mismo autor del documento, para las costas de Chile utilice material del siglo anterior, en especial los que en Inglaterra se conocieron en 1682 a raíz de la captura de galeón español Santo Rosario, con su valioso derrotero por parte de Sharp, y que significó la elaboración de copias como las de Basil Ringrose y las de William Hack ${ }^{40}$ En suma, tanto por la información geográfica contenida en el propio documento así como también porque corresponde a una tipología propia de la segunda mitad del siglo XVII, la datación final no pudo pasar de 1707 si se consideran las últimas anotaciones al texto desde 1703.

Ahora bien, en cuanto a la autoría, es imposible asignarle una firma específica a una persona, tomando en consideración de que la particularidad de dichos documentos es que correspondían a síntesis de información recogida de diversas fuentes, pero también es efectivo que todo piloto tenía su propio derrotero que corregía y mejoraba para las navegaciones futuras, por lo que el objetivo hasta la fecha ha sido dilucidar la posible pertenencia de tan importante documento. En sus primeras investigaciones, Ortiz Sotelo definió que el documento había pertenecido a la expedición de William Dampier, iniciada en 1703 y que había finalizado con su captura por parte de holandeses en el caribe en $1705{ }^{41}$ aunque no pudo resolver la hipótesis de la posible autoría o propiedad del mismo. Sin embargo, recientemente dicho autor, gracias al hallazgo de importante documentación relativa a la expedición, ha planteado una nueva hipótesis acerca de que el autor del derrotero en su versión original fuese el marino escocés Alexander Selkirk, piloto de la Cinque Ports, quien fue abandonado en 1704 en la isla Más a Tierra frente a la costa de Chile, sobreviviendo casi cinco años en el lugar. ${ }^{42}$ Ahora bien, cómo llegó el documento a Perú sigue siendo un mis-

40 Howse y Thrower, 1992. La captura es referida en la costa de Ecuador pero números autores hacen alusión de que el hecho ocurrió en Panamá.

41 Ortiz Sotelo, 2013. Sobre una biografía reciente de Dampier, véase Preston y Preston, 2010.

42 Ortiz Sotelo, 2013. 
terio sin resolver, aunque todo indica que perteneció a la referida nave perdida en las costas del virreinato.

En cuanto a la representación del estrecho de Magallanes sigue claramente la influencia del capitán Narborough, mejorando ostensiblemente la calidad cartográfica de los derroteros de Ringrose y Hack, quienes se basaron en una carta española de menor calidad. De allí que la idea de síntesis, propia de los derroteros de aquella época, se observe con claridad en beneficio de un mejor conocimiento de las costas y de una navegación más segura para los pilotos y de los cientos de vidas que estaban bajo su responsabilidad. Una prueba de esto es la información entregada en la foja anterior a la carta magallánica, en donde junto con representar la costa de la Patagonia occidental, refiere que

this Coast from the Island Guafo to Cape Victoria has been very Little frequented and much less the greatest part of Terra del fuego especially on S.W. side, therefore I have not been able to obtain any other description there of tan (which I take to be) the best General draft which I doubt is very imperfect and therefore the more care must be taken in Sailing on these Coast until better Discovery. ${ }^{43}$

Lo expuesto demuestra que para la confección de estas cartas náuticas, se requería una minuciosa búsqueda de antecedentes cartográficos y, cuando éstos no eran seguros o simplemente inexistentes, lo principal era evitar riesgos y navegar distantes de la costa desconocida. De hecho, el autor del documento estaba bastante bien informado sobre el conocimiento de la zona que comprendía la isla de Guafo, al sur del archipiélago de Chiloé, y el cabo Victoria, en la boca noroccidental del estrecho de Magallanes. Este problema era extensivo a los españoles y sólo pudo comenzar a ser correctamente explorado a fines del siglo XVIII y durante buena parte del XIX. De hecho, basta recordar las referidas exploraciones hispanas desde Chiloé a Magallanes, las que habían fracasado sólo unas pocas décadas antes de la confección del derrotero por la gran dificultad que generaba la navegación por los mares australes.

Respecto al estrecho, la descripción geográfica se realiza desde nororiente comenzando con el cabo Vírgenes, que el autor denomina Virgen María, siguiendo el error de Narborough. Posteriormente realiza un detallado relato acerca de la navegación en la referida boca oriental que, a la luz de los conocimientos de la época y comparados con los actuales, identifican

43 AHMGP, Fondo Derrotero Inglés, 44v. 


\section{EL ESTRECHO DE MAGALLANES COMO ANTESALA DEL PACÍFICO}

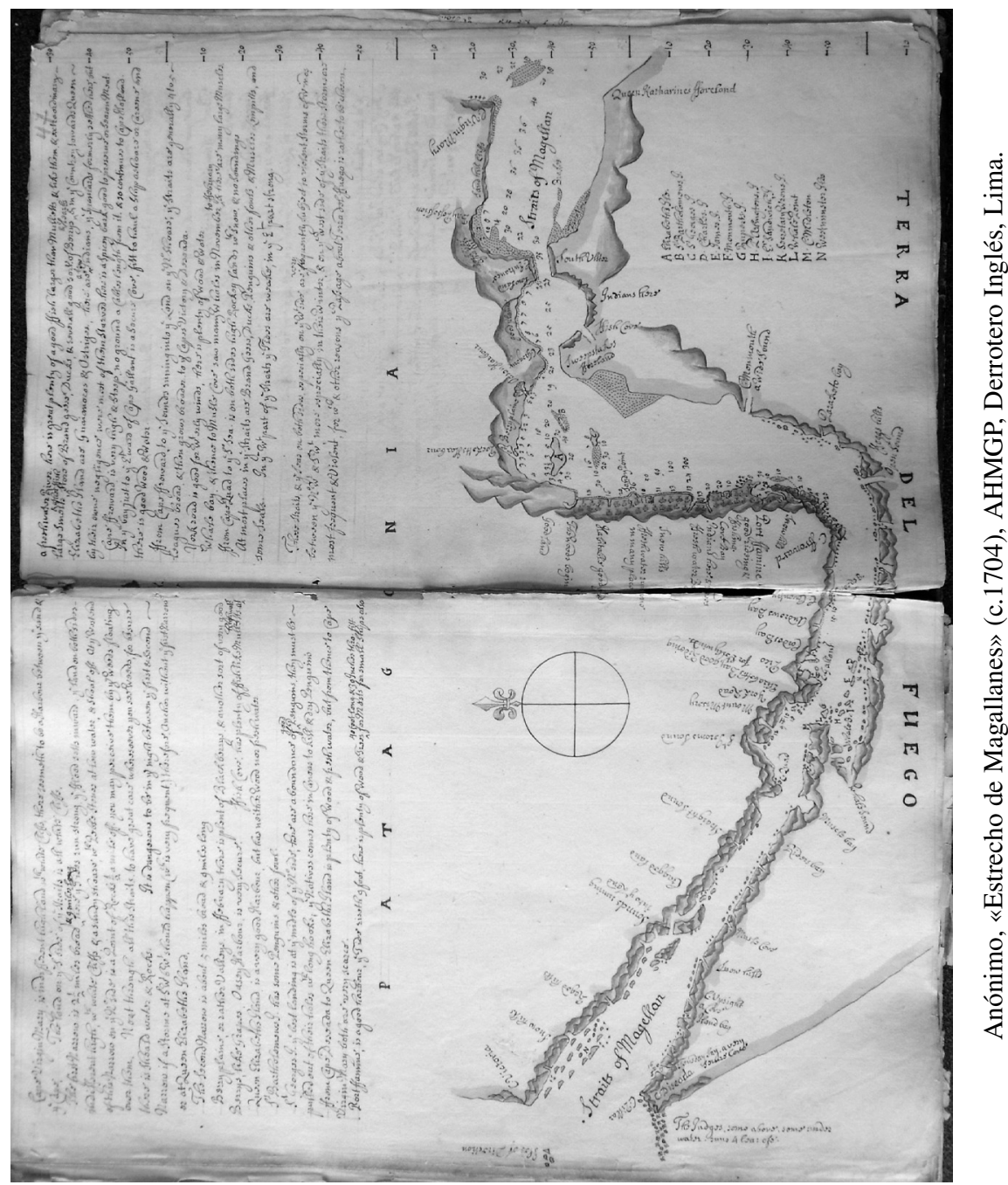


de manera bastante certera los peligros que significaba la navegación en dicho lugar. ${ }^{44} \mathrm{El}$ relato avanza al segundo y tercer estrecho en que se advierten los peligros de la navegación nocturna y de los fuertes vientos del suroeste, para lo cual se hace referencia a las posibilidades de sortear de buena forma el inconveniente, citando la isla Reina Isabel como un buen lugar donde fondear, aunque «Queens Elisabeth Island is a very good Harbour but has neither Wood nor fresh water». ${ }^{45}$

En cuanto a esta primera descripción, todos los sondajes que fueron realizados en la expedición de Narborough son replicados en la carta anónima pero resumiendo una buena parte concordante con la escala del mismo, sin embargo, son reproducidos con exactitud los bajos y otras referencias relevantes para la navegación. En cuanto a los topónimos, no hay sustanciales diferencias, confirmándose en gran medida el origen inglés de los mismos aunque con una variación importante: fuera de la toponimia específica del estrecho, no hay alusión a los territorios que le circundan, respetándose las clásicas denominaciones de Patagonia al norte y Terra del Fuego [sic] al sur, distanciándose de la denominación política contenida en la carta de Narborough, ya que a la Tierra del Fuego le suma el nombre de King Charles Southern Land, la concordancia con la edición impresa dedicada al monarca y la toma de posesión que, tal como se refirió, hizo el marino inglés en ambas costas del estrecho. El resto del documento vuelve a consolidar las principales referencias toponímicas del siglo XVI y las que pudo añadir Narborough en sus cartas manuscritas, así como también en la edición impresa de Thorton. Se hace alusión, por ejemplo, a las fuertes tormentas y vientos que hay en la parte occidental, lo que hace recomendable la navegación cercana a la costa de Tierra del Fuego. ${ }^{46}$

En suma, este derrotero manuscrito nos presenta un conocimiento acabado del estrecho de Magallanes, acorde a las últimas noticias que el autor pudo recabar para el buen cometido, de una navegación segura y fructífera. La expedición de Dampier y, específicamente, los pilotos que la componían enfrentaron el desafío de surcar el estrecho con las mejores herramientas del siglo, las cuales superaban con creces las que los bucaneros podían tener para ese mismo tiempo. De hecho, el derrotero de Ringrose en lo que se refiere al estrecho de Magallanes nos muestra que, hacia 1682, el conocimiento geográfico desde la perspectiva hispana era

44 AHMGP, Fondo Derrotero Inglés, 46v.

45 AHMGP, Fondo Derrotero Inglés, 46v.

46 AHMGP, Fondo Derrotero Inglés, 47. 
precario, muy esquemático y con gran ausencia de información. En cambio, el referido derrotero inglés, conservado en Lima, es la prueba empírica de que estos documentos eran la síntesis de largas experiencias náuticas que habían incorporado el mejor trabajo realizado hasta entonces: la exploración de John Narborough, quien a su vez unía todo el conocimiento que se remontaba hasta el siglo XVI. ${ }^{47}$

Recibido el 3 de septiembre de 2013 Aceptado el 17 de octubre de 2013

\section{Bibliografía}

Alcedo, Antonio de: Diccionario geográfico-histórico de las Indias Occidentales, t. IV, Madrid, Imprenta de Manuel González, 1789.

Barros, José Miguel: «Primer testimonio de Tomé Hernández sobre las fundaciones hispánicas del Estrecho de Magallanes», Anales Instituto de la Patagonia (AIP), 9, 1978, 65-75.

Bradley, Peter T.: Navegantes Británicos, Madrid, Mapfre, 1992.

Cabrera, Ana María y Favero, Amalia del: «Toponimia histórica de la Costa Nororiental del Estrecho de Magallanes», AIP, 14, 1983, 13-42.

Cummins, Francis: Francis Drake, London, Weindenfeld \& Nicolson, London, 1995.

Espinoza, Juan Pablo y Espinoza, Ricardo: «La increíble empresa de Sarmiento de Gamboa y su triste fin: Posibles causas de la tragedia en el Estrecho de Magallanes en el siglo XVI», Revista Médica de Chile, 138, 2010, 1456-1460.

Fletcher, Francis: The World encompassed by sir Francis Drake, London, Nicholas Bourne, 1628.

Guarda, Gabriel: Atlas Cartográfico del Reino de Chile, Siglos XVIII-XIX, Santiago de Chile, Instituto Geográfico Militar, 1981.

Guarda, Gabriel y Moreno Jeria, Rodrigo: Monumenta Cartographica Chiloensia, Santiago de Chile, Corporación Patrimonio Cultural de Chile, Santiago de Chile, 2008.

Guarda, Gabriel y Moreno Jeria, Rodrigo: Monumenta Cartographica Valdiviensae, Santiago de Chile, Corporación Patrimonio Cultural de Chile, Santiago de Chile, 2010.

Howse, Derek y Thrower, Norman J.W. (ed.): A Buccanneer's Atlas, Basil Ringrose's South Sea Waggoner, Los Angeles, University of California Press, 1992.

47 Sinceros agradecimientos a Juan y Peggy Rada por haber permitido trabajar al autor con su valiosa colección cartográfica. 
Latorre, Guillermo: «Sustrato y superestrato multilingües en la toponimia del extremo sur de Chile», Estudios Filológicos, 33, 1998, 55-67.

Martin Merás, Luisa: Cartografía Marítima Hispana, Barcelona, Lunwerg - CSIC, 1993.

Martinic, Mateo y Moore, David: «Las Exploraciones Inglesas en el Estrecho de Magallanes 1670-1671. El Mapa Manuscrito de John Narborough», AIP, 13, 1982, 7-20.

Martinic, Mateo: «Bahía Cordes-Fortescue (Estrecho de Magallanes), su ocupación por holandeses en 1599 y franceses en 1694-1699», Boletín de la Academia Chilena de Historia, 65-66, 1998-1999, 307-320.

Martinic, Mateo: Cartografía Magallánica, Punta Arenas, Universidad de Magallanes, 1999.

Martinic, Mateo: «Los holandeses en las Islas de los Pingüinos (1599-1615)», Magallania, 40 (2), 2012, 7-22.

Medina, José Toribio: Ensayo acerca de una mapoteca chilena ó sea de una colección de los títulos de los mapas, planos y vistas relativos á Chile arreglados cronológicamente, con una introducción histórica acerca de la geografía y cartografía del país, Santiago de Chile, Imprenta Ercilla, 1889.

Nodal, Bartolomé García de y Nodal, Gonzalo de: Relación del viaje que por orden de su Magestad y acuerdo del Real Consejo hicieron los capitanes Bartolomé García de Nodal y Gonzalo de Nodal, hermanos, naturales de Pontevedra, al descubrimiento del Estrecho Nuevo de San Vicente y reconocimiento del de Magallanes, Madrid, impreso por Fernando Correa de Montenegro, 1621.

Ortiz Sotelo, Jorge: Un derrotero Inglés de las Costas de América (1703-1704), Lima, Dirección General de Intereses Marítimos, 1988.

Ortiz Sotelo, Jorge: Derrotero General del Mar del Sur, del capitán Pedro Hurtado de Mendoza, hecha por el capitán Manuel Joseph Hurtado en el Puerto del Callao, año de 1730, Lima, Fondo de Publicaciones Dirección de Intereses Marítimos, 1993.

Ortiz Sotelo, Jorge: «La expedición de Dampier y un derrotero inglés en Perú», Sagredo Baeza, Rafael, Moreno Jeria, Rodrigo (eds.), Del Mar del Sur al Pacífico, Santiago de Chile, Editorial Universitaria, Biblioteca Nacional de Chile, Universidad Adolfo Ibáñez, 2013.

Pigafetta, Antonio: Primer Viaje en torno al Globo, Madrid, Espasa Calpe, 1922.

Preston, Diana y Preston, Michael: A Pirate Of Exquisite Mind: The Life Of William Dampier, London, Corgi Books, 2010.

Rosenblitt, Jaime y Sanhueza, María Carolina: Cartografía Histórica de Chile, Santiago de Chile, Cámara Chilena de la Construcción, Pontificia Universidad Católica de Chile, Dirección de Bibliotecas Archivos y Museos, 2010.

Sarmiento de Gamboa, Pedro: Viage al Estrecho de Magallanes, Madrid, Imprenta Real de la Gazeta, Madrid, 1768. 
Seixas y Lobera, Francisco: Descripción Geographica y Derrotero de la Región Austral Magallánica, Madrid, Imprenta Antonio de Zafra, 1690.

Steward Stoke, Hamish y Cabello, Claudia: «Los Británicos en Magallanes 15501800», AIP, 28, 2000, 15-27.

Vargas y Ponce, José: Relación del último viage al Estrecho de Magallanes de la fragata de S.M Santa María de la Cabeza en los años de 1785 y 1786. Extracto de todos los anteriores desde su descubrimiento, impresos y MSS. y noticias de los habitantes, suelo, clima, y producciones del Estrecho, Madrid, Impreso por la viuda de Ibarra, hijos y Compañía, 1788.

Vásquez, Nelson, Iglesias, Ricardo y Molina, Mauricio: Cartografía Histórica de Valparaíso, Valparaíso, Ediciones Universitarias de Valparaíso, 1999.

Wallis, Helen: «English Enterprise in the Region of the Strait of Magellan» en Parker, John (edit.), Merchants and Scholars, Minneapolis, University of Minnesota Press, 1965, 193-220. 